\title{
CARACTERIZACIÓN DE FRUTAS COMESTIBLES DE ESPECIES NATIVAS DE USO POPULAR EN EL PARQUE PROVINCIAL "SALTO ENCANTADO DEL VALLE DEL CUÑÁ PIRÚ - MISIONES”
}

\author{
NATALIA PINTALUBA ${ }^{1}$ \& PAULA ALAYÓN LUACES ${ }^{2}$
}

\begin{abstract}
Summary: Pintaluba, N. \& P. Alayón Luaces. 2013. Characterization of edible fruit from native species of popular use in the Provincial Park "Salto Encantado del Valle de Cuñá Pirú - Misiones". Bonplandia 22(2): 191-201.
\end{abstract}

\begin{abstract}
Misiones Province is located in northeastern Argentina. Its native flora has several species little studied, that have potential for fresh consumption, candy industry and medicinal, but still is lacking of information for the use of these species. The Nature Reserve Salto Encantado del Valle del Cuñá Piru is an area untouched by man and where the characteristics of the native species are retained. The Provincial Park has an area of 13,227 ha so it has been selected three representative monitoring sites, located at different altitudes. The common feature between sites was that they are at the edge of the mainstream of Cuñá Piru creek, which runs through the park. The data were relieved from all species of fruit importance that were in the selected sites, phenological records were performed and the fruits were harvested for performing physicochemical analysis of quality. A diversity of native species that are part of the flora of the province, with great potential for the use of its fruits for fresh consumption has been detected. This is the first report of the physical and chemical characteristics of the native fruits that grow on the banks of Arroyo Cuñá Piru, Misiones province, Argentina.
\end{abstract}

Keywords: Jungle missionary, fruit, acidity, sugars.

Resumen: Pintaluba, N. \& P. Alayón Luaces. 2013. Caracterización de frutas comestibles de especies nativas de uso popular en el Parque Provincial "Salto Encantado del Valle de Cuñá Pirú - Misiones". Bonplandia 22(2): 191-201.

La provincia de Misiones se encuentra ubicada al noreste de la República Argentina. Su flora nativa presenta diversas especies, poco estudiadas, cuyos frutos tienen potencial utilización para consumo en fresco, industria de dulces, como medicinales, entre otros sin embargo aún falta información para el aprovechamiento de estas especies. La Reserva Natural Salto Encantado del Valle de Cuñá Pirú es un área no modificada por el hombre y donde se mantienen las características propias de las especies nativas, el Parque Provincial tiene una extensión de 13.227 has. por lo que se seleccionaron tres sitios de seguimiento representativos del mismo, ubicados a diferentes altitudes. La característica común entre los sitios fue que éstos están a la vera del arroyo principal de la reserva que es el Cuñá Pirú, el cual corre encajonado atravesando el parque. Se relevaron datos de la totalidad de especies de importancia frutícola que se

${ }^{1}$ Alumna, Facultad de Ciencias Agrarias, Universidad Nacional del Nordeste. Trabajo final de graduación modalidad tesina.

${ }^{2}$ Docente, Cátedra de Fruticultura, Facultad de Ciencias Agrarias, Universidad Nacional del Nordeste. Sargento Cabral 2131, Corrientes, Argentina. palayonluaces@yahoo.com 


\begin{abstract}
encontraban en los sitios seleccionados, se realizaron seguimientos fenológicos de los mismos y se recolectaron los frutos para la realización de análisis físico-químico de calidad de fruta. Se detectaron una gran diversidad de especies nativas que son parte de la flora de la provincia, con un gran potencial para el aprovechamiento de sus frutas para consumo en fresco. Este es el primer reporte de las características físico químicas de los frutos nativos que crecen a la vera del Arroyo Cuña Pirú, provincia de Misiones, Argentina.
\end{abstract}

Palabras clave: Selva misionera, frutales, acidez, azúcares.

\section{Introducción}

El Parque Provincial Salto Encantado del Valle del Cuñá Pirú presenta una gran biodiversidad nativa en su flora y su fauna. Se encuentran estudios realizados por la Fundación Vida Silvestre Argentina en el año 1990 (Bertolini, 1999) que cita la existencia de 72 especies pertenecientes a la flora nativa, también se citan estudios de algas del Arroyo Cuñá Pirú y sus afluentes (UNLP, 1997); estudios de Pteridofitas nativas (Márquez \& al., 2006) inclusive se encuentran trabajos detallados de mamíferos (Cirignoli \& al., 2011).

Por medio de entrevistas con gente idónea y pobladores de la región se manifiesta la presencia de especies nativas que por el saber popular sus frutas serían valiosas para el consumo en fresco y elaboración de dulces.

Sin embargo, no encontramos estudios exhaustivos que caractericen las especies nativas con valor frutícola que allí existen, por lo tanto se desconoce su potencial uso y aprovechamiento. Más aún, se conoce de la incidencia que tienen las diferentes altitudes sobre las características morfo-anatómicas y fisiológicas de las especies vegetales en general, pero no encontramos citas que nos brinden información del efecto del relieve sobre las características de estas especies.

El objetivo del presente trabajo fue realizar el reconocimiento, la identificación y la caracterización de las frutas de especies nativas con potencial valor frutícola, ubicadas a diferentes altitudes dentro del Parque Provincial Salto Encantado del Valle del Cuñá Pirú, Misiones.

\section{Material y Métodos}

\section{Características del sitio de estudio}

El estudio se realizó en el Parque Provincial Salto Encantado del Valle del Cuñá PirúMisiones (PPSEV Cuñá-Pirú) ubicado en el centro de la provincia de Misiones, entre los meridianos 5449'45" O y 5458'39' O y los paralelos $26^{\circ} 5^{\prime} 48^{\prime \prime}$ S y $27^{\circ} 5^{\prime} 40^{\prime \prime}$ S, a unos 170 km de la ciudad de Posadas (Bertolini, 1999) (Fig. 1).

De acuerdo al relevamiento de suelos de la provincia de Misiones (Ligier, 1990) los sitios de estudio presentan una asociación de suelos de los tipos: Hapludoles énticos, Eutocreptes dístricos y Udortentes típicos se caracterizan por presentarse en sectores con pendientes dominantes del 20 al 40\%. Los horizontes orgánicos están constituidos por restos de hojarasca en distintos estados de descomposición que normalmente no superan los $10 \mathrm{~cm}$ y pueden estar ausentes en sectores de selva muy degradada. Son suelos bien drenados, someros o moderadamente profundos. De severa a moderada pedregosidad, evidencian una muy alta susceptibilidad a la erosión hídrica.

En relación a las características hidrológicas del Parque, el arroyo Cuñá-Pirú desagua en el río Paraná, por lo que él y sus afluentes pertenecen a dicha cuenca. En el límite Este del Parque Salto Encantado, el arroyo Cuñá-Pirú forma un salto denominado "Salto Encantado", que se destaca por sus $60 \mathrm{~m}$ de altura, dándole nombre al Parque. Numerosos cursos de agua menores recorren el Parque, formando pequeñas cascadas.

El clima de Misiones es subtropical húmedo sin estación seca. La localidad de Aristóbulo 


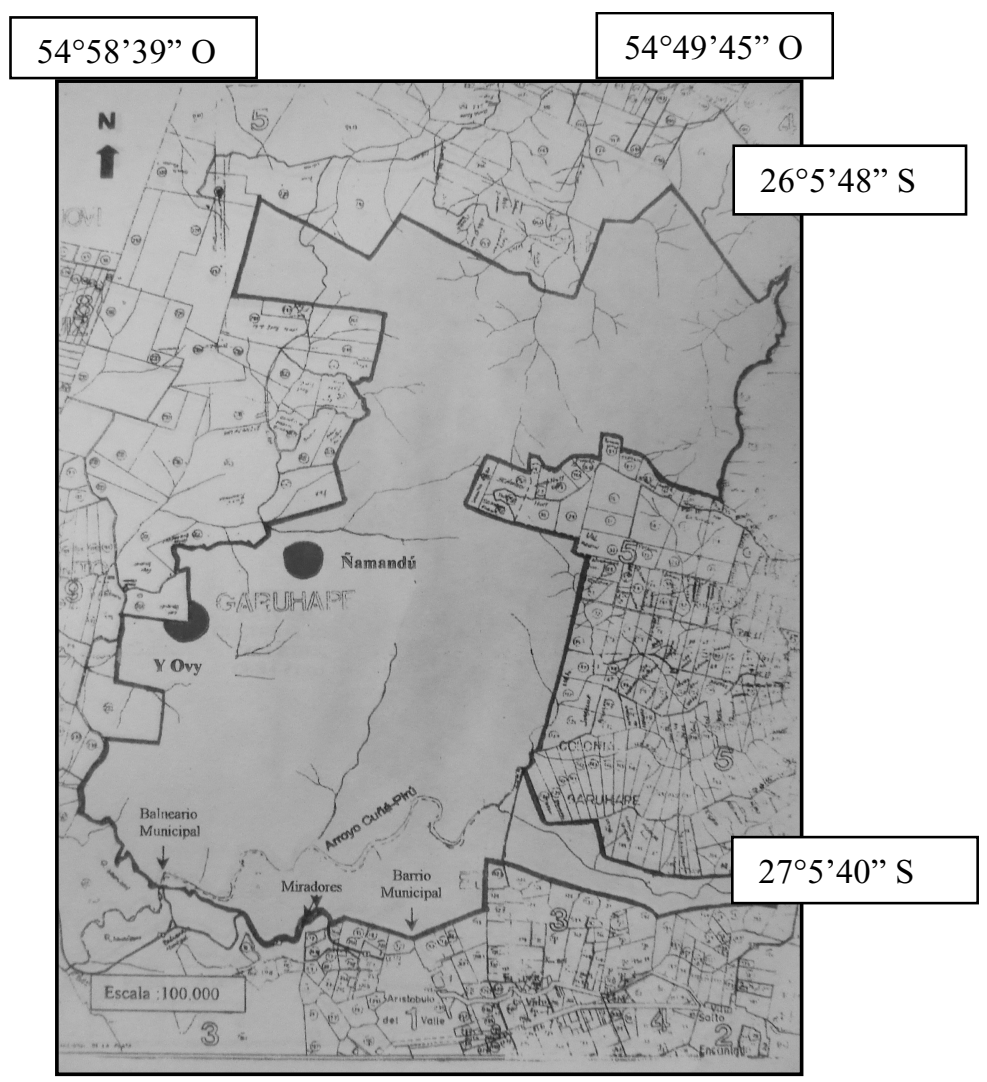

Fig. 1. Mapa del Parque Provincial Salto Encantado del Valle del Cuñá Pirú, Misiones.

del Valle, lugar representativo del PPSEV Cuñá-Pirú, presenta una temperatura media en el mes de enero de $23,8^{\circ} \mathrm{C}$ y en el mes de julio de $13,6^{\circ} \mathrm{C}$, con una amplitud térmica diaria alta por efecto de la altitud y la topografía, la precipitación media anual es de $1831 \mathrm{~mm}$ (Margalot, 1985).

\section{Selección de los sitios de muestreo}

Para delimitar el área de trabajo y de reconocimiento se tomó como referencia el principal afluente de la Reserva Natural, el arroyo Cuñá Pirú el cual atraviesa la totalidad del Parque. A lo largo del arroyo se reconocen diferentes altitudes, de sustancial magnitud, las cuales fueron tomadas en cuenta para la selección final de los sitios.

Se seleccionaron tres sitios en diferentes altitudes, los cuales se encuentran a la vera del arroyo y cuyas dimensiones son de 125 $\mathrm{m}$ de largo bordeando el mismo y de 75 $\mathrm{m}$ de ancho hacia el interior de la selva, delimitando un área de $9000 \mathrm{~m}^{2}$ para cada sitio (Fig. 2).

Sitio A: Balneario Municipal de Aristóbulo del Valle - Misiones (200 m s.n.m.)

Sitio B: Salto Encantado - Misiones (420 m s.n.m.)

Sitio C: Salto Encantado - Misiones (360 m s.n.m.)

\section{Marcación y reconocimiento de especies}

Una vez establecidos y delimitados los sitios de trabajo, se procedió al reconocimiento y marcación de especies nativas con valor frutícola que se encontraban dentro de los mismos. Se hicieron recuentos de la totalidad de ejemplares en cada sitio previamente definido. Luego de la marcación de las especies, se midieron los diámetros de tronco de cada ejemplar a la altura de pecho y se expresó en centímetros. 

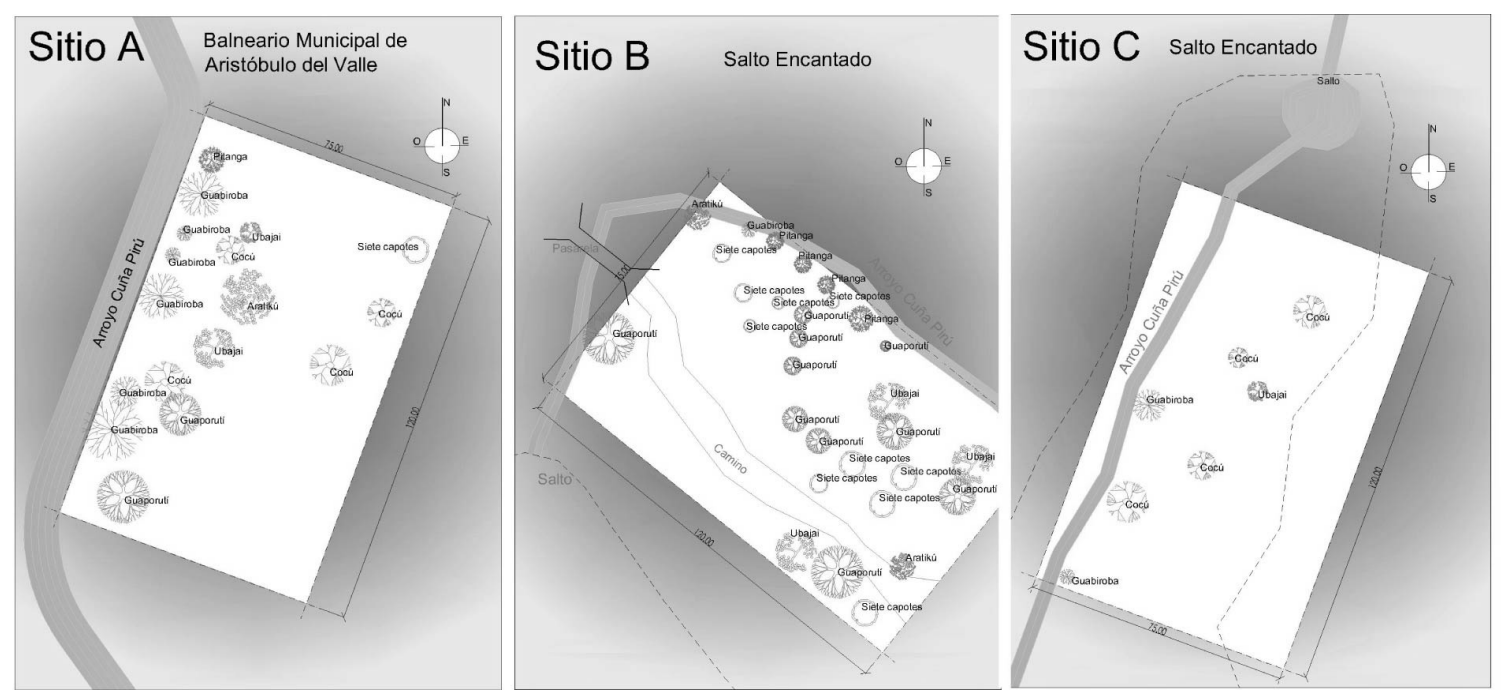

Fig. 2. Localización de los Sitios de estudio respecto al arroyo Cuñá Pirú y altura sobre el nivel del mar (m s.n.m.). Sitio A: 200 m s.n.m., Sitio B: 420 m s.n.m. y Sitio C: 360 m s.n.m. Parque Provincial Salto Encantado del Valle del Cuñá Pirú, Misiones, Argentina.

\section{Registros fenológicos}

Quincenalmente, se realizaron determinaciones fenológicas recorriendo todos los sitios de muestreo. Por la gran variabilidad de especies en estudio, se establecieron como momentos fenológicos comunes a todas las especies: prefloración, floración, crecimiento de frutos y momento de cosecha. Los estadios fenológicos registrados de cada especie fueron volcadas en planillas preparadas para tal fin, iniciando los registros en el mes de julio de 2012 y finalizando en abril de 2013.

\section{Recolección y análisis de calidad de frutas}

El proceso de recolección de frutas se realizó con la ayuda de escalera y pértiga extensible, debido a la gran altura de los ejemplares y la cosecha consistió en el corte de porciones de ramas con frutas, con cantidades variables dependiendo de la especie.

Las variables de calidad físico química de las frutas están directamente relacionadas a las características propias de cada una de las especies en estudio por ello parte del análisis de este trabajo fue establecer dichas particularidades. Dependiendo de las variables genotípicas y fenotípicas de cada especie se analizaron los siguientes parámetros:
- Peso fresco de las frutas: se pesaron individualmente las frutas en gramos $(\mathrm{g})$ con balanza de precisión.

- Peso seco de las frutas: las frutas se llevaron a estufa a $70^{\circ} \mathrm{C}$ hasta peso constante y se expresó el resultado en gramos.

- Forma de las frutas: se caracterizó cada especie en particular.

- Dimensiones de las frutas: se midieron los diámetros de las frutas en $\mathrm{mm}$ con calibre digital para poder obtener un promedio de las mismas e inferir en su forma.

Para el análisis de la calidad físico química se analizó:

- Coloración: Se estableció una escala de presencia de color en el fruto para poder clasificarlos y evaluarlos. La escala propuesta fue:

a. Cubiertos: frutos cuya epidermis estaba totalmente cubierta por la tonalidad característica de la especie.

b. Pintones: frutos cuya epidermis parcialmente cubierta de tonos verdes entremezclados con la tonalidad típica de la especie.

c. Verdes: frutos en cuya epidermis sólo se apreció una coloración verde.

- Sólidos solubles: se evaluó el contenido de sólidos solubles utilizando un refractómetro 
manual Arcano 0-32 $2^{\circ}$ expresados en ${ }^{\circ}$ Brix. - Acidez titulable: se determinó la acidez por titulación con $\mathrm{NaOH} 0,1 \mathrm{~N}$ y fenolftaleína $1 \%$. El jugo de fruta se filtró y se diluyó en una relación jugo:agua destilada de 1:10. Los resultados se expresan en \% de ácido cítrico.

\section{Recolección de ejemplares para herbario}

Con el propósito de disponer de ejemplares de herbario para material de consulta de las especies nativas estudiadas se recolectaron hojas, flores o frutos. Los ejemplares herborizados fueron llevados al Instituto de Botánica del Nordeste (IBONE) para su clasificación botánica y su conservación.

\section{Resultados y Discusión}

Las características de los sitios de estudio fueron particularmente diferentes. El sitio A se caracterizó por ser más tupido que los otros dos, ya que se observaron una gran cantidad de especies típicas de la Selva Misionera y en especial la presencia de bambúseas que dificultan el acceso a esta porción de la selva. El sitio B, a diferencia del sitio A, presentó un menor grado de tupidez debido al mantenimiento y limpieza necesaria por la ubicación particular del mismo. El sitio $\mathrm{C}$ se trató del lugar más húmedo y rocoso, se caracterizó por tener poca entrada de luz aprovechable para las plantas, debido a que se encuentra al pie de la sierra acompañando al arroyo que va encajonado en este relieve.

Se observaron gran diversidad de especies frutícolas a la vera del arroyo Cuñá Pirú en los tres sitios evaluados, la presencia diferencial en lo que respecta a las especies de los tres sitios se muestran en la Tabla 1.

Tabla 1. Especies nativas de importancia frutícola encontradas en los tres sitios de muestreo. A: 200 m s.n.m.;

B: 420 m s.n.m.; C: 360 m s.n.m.

\begin{tabular}{llcccc}
\hline \multicolumn{1}{c}{ Nombre vulgar } & \multicolumn{1}{c}{ Nombre científico } & Familia & $\begin{array}{c}\text { Sitio } \\
\text { A }\end{array}$ & $\begin{array}{c}\text { Sitio } \\
\text { B }\end{array}$ & $\begin{array}{c}\text { Sitio } \\
\text { C }\end{array}$ \\
\hline Guabiroba & Campomanesia xanthocarpa Mart. ex O. Berg & Mirtáceas & 6 & 1 & 2 \\
Ubajai & Eugenia pyriformis Camb. & Mirtáceas & 2 & 4 & 1 \\
Guaporutí & Plinia rivularis (Cambess.) Rotman & Mirtáceas & 2 & 10 & ------ \\
Siete Capotes & Campomanesia guazumaefolia Blume & Mirtáceas & 1 & 10 & ------- \\
Aratikú & Rollinia emarginata Schltdl. & Anonáceas & 2 & 2 & ------ \\
Cocú & Allophylus edulis (St. Hil.) Radlkofer & Sapindáceas & 4 & ------ & 4 \\
Pitanga & Eugenia uniflora L. & Mirtáceas & 1 & 4 & ------ \\
Yacaratiá & Jacaratia spinosa (Aubl.) A. DC & Caricáceas & ------ & 1 & ------ \\
\hline \multicolumn{2}{l}{ Total de especies de interés frutícola por sitio } & & $\mathbf{1 8}$ & $\mathbf{3 2}$ & $\mathbf{7}$ \\
\hline
\end{tabular}

\section{Sitio A}

En el Sitio A (200 m s.n.m.) ubicado en el Balneario Municipal de la localidad de Aristóbulo del Valle se observó una totalidad de dieciocho ejemplares pertenecientes a cinco géneros diferentes y tres familias. En relación a la cantidad de individuos encontrados el Guabiroba (Campomanesia xanthocarpa) es predominante en este sitio con seis ejemplares de los dieciocho de importancia frutícola encontrados. Por su parte se encontraron cuatro ejemplares de Cocú (Allophylus edulis). El Guaporutí (Plinia rivularis) y Aratikú (Rollinia emarginata) estuvieron presentes con dos ejemplares cada uno al igual que el Ubajai (Eugenia pyriformis) $\mathrm{y}$ en menor medida con un solo ejemplar se encontró al Siete Capotes (Campomanesia guazumaefolia) y la Pitanga (Eugenia uniflora). 


\section{Sitio B}

E1 Sitio B, está ubicado a 420 m s.n.m. en el Salto Encantado, siendo el de mayor altitud de los tres sitios de muestreo. En este sitio toman importancia en presencia las especies del Siete Capotes y Guaporutí con diez ejemplares cada uno. Se encontraron cuatro plantas de Pitanga y cuatro de Ubajai, dos ejemplares de Aratikú y un único ejemplar de Guabiroba y de Yacaratiá (Jacaratia spinosa). El Cocú no se encontró en esta zona de estudio. Se contabilizó un total de treinta y dos plantas de siete géneros distintos siendo el sitio que más ejemplares tuvo con una gran variabilidad en tamaño y distribución.

\section{Sitio C}

El Sitio C está ubicado a 360 m s.n.m. aproximadamente y se encuentra al pie de la cascada que forma el arroyo Cuña Pirú del Salto Encantado (atractivo turístico conocido como Salto Encantado). En este sitio se encontraron cuatro ejemplares de Cocú, dos Guabiroba y un solo individuo de Ubajai totalizando siete árboles en estudio de tres géneros diferentes, sin embargo no se hallaron ejemplares de Guaporutí, Siete Capotes, Pitanga, Yacaratiá y Aratikú.
Respecto al tamaño de los árboles, se observó en los tres sitios de muestreo una gran heterogeneidad en relación al diámetro de troncos de las especies nativas frutícolas (Tabla 2).

En el sitio A se observó que los ejemplares en estudio fueron variados en lo que respecta a su diámetro. En general los árboles que se encontraron en este sitio presentaron un gran porte en algunos de los ejemplares de especies como el Guaporutí, Cocú y Guabiroba.

Los ejemplares del sitio B presentaron tamaños muy variables detectándose presencia tanto de renovales como de árboles de gran porte e intermedios.

El caso particular del Yacaratiá se destacó por ser un ejemplar de gran porte y fue el que mayor diámetro de tronco presentó.

En el sitio $\mathrm{C}$ los árboles que se encontraron fueron de escasa magnitud y casi en su totalidad en mal estado sanitario probablemente favorecidas por las condiciones de alta humedad relativa a las que están expuestas estos ejemplares.

\section{Registros de estados fenológicos}

Los estados fenológicos de las especies en los diferentes sitios presentaron un comportamiento

Tabla 2. Diámetros de tronco a la altura de pecho de los ejemplares nativos de cada sitio de evaluación expresados en $\mathrm{cm}$. Las barras inclinadas separan ejemplares.

\begin{tabular}{cccc}
\hline Especies frutícolas nativas & SITIO A & SITIO B & SITIO C \\
\hline Aratikú & 12,90 & $10,19 / 15,92$ & \\
Cocú & $15,13 / 3,69$ & & $7,64 / 13,69$ \\
& $10,51 / 11,46$ & & \\
Guaporutí & $41,40 / 25,80$ & $2,87 / 7 / 7,37,32 / 13,69 /$ & \\
& & $11,78 / 8,76 / 19,90 / 16,08$ & \\
Guabiroba & $40,76 / 6,37 / 14,01 / 5,09$ & $15,13 / 36,30$ & \\
& $3,50 / 17,83$ & 1,75 & \\
Siete Capotes & 5,73 & $8,60 / 14,65,73$ & \\
& & $28,66 / 5,70 / 14,01 / 10,51 / 10,60$ & \\
Pitanga & 5,09 & $16,72 / 14,17$ & 2,87 \\
Ubajai & $7,80 / 4,46$ & $6,05 / 9,88 / 12,10 / 11,78$ & \\
Yacaratiá & & $3,18 / 21,17 / 25,95 / 17,83$ & \\
\hline
\end{tabular}


similar, en Tabla 3 se indican los registros de las mismas, sin diferenciar los sitios.

Considerando la totalidad de las especies se observó que el período reproductivo, desde floración a cosecha, se extiende desde los meses de septiembre a febrero.

Los primeros frutos recolectados fueron de las especies de Guabiroba y Guaporutí, ambos cosechadas en el mes de noviembre, coincidente con lo citado por Lopez \& al. (1987).

Respecto del Cocú se encontraron coincidentemente flores y frutos verdes en el mes de septiembre y se observó que esta especie presenta una floración continua.

El Ubajai tuvo un período de floración algo más amplio (desde noviembre hasta diciembre) y los frutos se recolectaron a mediados del mes de enero. Mientras que el Siete Capotes floreció entre los meses de noviembre a diciembre, y la fructificación se extendió hasta febrero.

Tanto el Yacaratiá y Aratikú sus frutos fueron cosechados en el mes de febrero lo cual coincide con lo mencionado por Lopez \& al. (1987) para estas especies.

En la mayoría de las especies encontradas, el período de floración comprende una amplitud de entre uno y dos meses. Sin embargo el período de fructificación (crecimiento y maduración de los frutos) es acotado y concentrado en la mayoría de los casos (menor a 30 días), por lo que fue necesario realizar monitoreos constantes para poder recolectar los frutos ya que los mismos no son retenidos por un largo período en las plantas. La especie conocida como Siete
Capotes presentó otro comportamiento ya que sus frutos permanecen en la planta por más tiempo (entre 30 y 45 días).

\section{Análisis físico químico de los frutos}

Se analizaron los frutos de las especies Guabiroba, Guaporutí, Siete Capotes, Aratikú, Ubajai y Yacaratiá recolectados en los sitios A y B.

En la Tabla 4, se detallan las especies evaluadas de cada uno de los sitios, el momento de recolección, coloración, peso fresco promedio por fruto en gramos, peso seco promedio por fruto en gramos, tamaño en $\mathrm{mm}$, contenido de sólidos solubles ( $\left.{ }^{\circ} \mathrm{Brix}\right)$ y acidez expresada como \% de ácido cítrico.

\section{Sitio A}

De los dieciocho ejemplares encontrados en el sitio A (pertenecientes a cinco géneros diferentes) se cosecharon frutas de cuatro de ellos, correspondientes a las especies nativas del Guabiroba, Guaporutí, Siete capotes y Aratikú.

Los frutos del Guabiroba cosechados en Noviembre de 2012 presentaron un tamaño promedio de $16,86 \mathrm{~mm}$ y un peso promedio por fruto de $2,70 \mathrm{~g}$ y totalmente cubiertos por la coloración amarilla intensa característica (Ortega Torres \& al., 1989). De la relación PS/ PF surge que estos frutos tienen en promedio un $81,48 \%$ de agua en el fruto. En cuanto al contenido de sólidos solubles presentaron un $16,4^{\circ}$ Brix y una acidez de $1,08 \%$.

Tabla 3. Registros fenológicos de las especies encontradas en los tres sitios.

\begin{tabular}{|c|c|c|c|c|c|c|c|c|c|c|}
\hline \multirow{3}{*}{ Especies } & \multicolumn{10}{|c|}{ Período de floración a cosecha } \\
\hline & \multicolumn{6}{|c|}{2012} & \multicolumn{4}{|c|}{2013} \\
\hline & jul & ago & sept & oct & nov & dic & ene & feb & mar & $\mathrm{abr}$ \\
\hline Aratikú & & & & & & * & $*$ & $*$ & & \\
\hline Guabiroba & & & & $*$ & $*$ & & & & & \\
\hline Guaporutí & & & & $*$ & $*$ & & & & & \\
\hline Cocú & & & $*$ & $*$ & $*$ & & & & & \\
\hline Siete capotes & & & & & $*$ & $*$ & $*$ & $*$ & & \\
\hline Ubajai & & & & $*$ & $*$ & $*$ & $*$ & & & \\
\hline Yacaratiá & & & & & & $*$ & $*$ & $*$ & & \\
\hline
\end{tabular}


Tabla 4. Análisis físico-químico realizados a los frutos de las especies nativas encontradas.

\begin{tabular}{|c|c|c|c|c|c|c|c|c|c|}
\hline$\stackrel{0}{\vec{n}}$ & Especie & $\begin{array}{l}\text { Fecha de } \\
\text { cosecha }\end{array}$ & Color & $\begin{array}{c}\text { PF } \\
\text { fruto } \\
(g)\end{array}$ & $\begin{array}{c}\text { PS } \\
\text { fruto } \\
\text { (g) }\end{array}$ & $\begin{array}{c}\% \mathrm{H}_{2} \mathrm{O} \\
\text { en el } \\
\text { fruto }\end{array}$ & $\begin{array}{c}\text { Tamaño } \\
\text { (mm) }\end{array}$ & $\begin{array}{c}\text { Sólidos } \\
\text { solubles } \\
\left({ }^{\circ} \text { Brix) }\right.\end{array}$ & $\begin{array}{c}\text { Acidez } \\
\text { (\%) }\end{array}$ \\
\hline \multirow{7}{*}{$\mathbf{A}$} & Guabiroba & $09 / 11 / 12$ & Cubiertos & 2,70 & 0,50 & 81,48 & 16,86 & 16,4 & 1,08 \\
\hline & Guaporutí & $12 / 11 / 12$ & Cubiertos & 2,40 & 0,41 & 82 & 15,55 & 17,8 & 0,29 \\
\hline & \multirow{3}{*}{ Siete Capotes } & \multirow{3}{*}{$22 / 01 / 13$} & Pintones & 2,00 & 0,27 & 86 & 13,88 & 11,9 & 0,12 \\
\hline & & & Maduros & 7,70 & 1,60 & 80,52 & 25,20 & 14,75 & 4,40 \\
\hline & & & Inmaduros & 5,20 & 1,50 & 69,24 & 25,58 & 12,5 & 3,74 \\
\hline & \multirow[t]{2}{*}{ Aratikú } & \multirow[t]{2}{*}{$25 / 02 / 13$} & Cubiertos & 29,5 & ---- & --- & 37,97 & 19,5 & 0,33 \\
\hline & & & Verdes & 31,0 & 10,05 & 67,58 & 38,45 & $\mathrm{~s} / \mathrm{d}$ & $\mathrm{s} / \mathrm{d}$ \\
\hline \multirow{8}{*}{ B } & \multirow[t]{2}{*}{ Guaporutí } & $09 / 11 / 12$ & Cubiertos & 1,40 & 0,30 & 78,58 & 14,28 & 10,6 & 0,25 \\
\hline & & & $\begin{array}{l}\text { Verdes y } \\
\text { pintones }\end{array}$ & 1,30 & 0,28 & 78,47 & 13,33 & 8 & 0,25 \\
\hline & Ubajai & $14 / 01 / 13$ & Cubiertos & 4,75 & 0,75 & 85 & 19,54 & 8,2 & 3,78 \\
\hline & Siete Capotes & $22 / 01 / 13$ & Maduros & 12,0 & 2,50 & 79,17 & 30,30 & 13,8 & 5,82 \\
\hline & & & Inmaduros & 5,80 & 1,80 & 68,97 & 29,90 & 13 & 4,95 \\
\hline & \multirow[t]{2}{*}{ Aratikú } & $25 / 02 / 13$ & Cubiertos & 69,0 & ---- & ---- & 46,80 & 19 & 0,25 \\
\hline & & & Verdes & 49,5 & 16,32 & 67 & 45,80 & $\mathrm{~s} / \mathrm{d}$ & $\mathrm{s} / \mathrm{d}$ \\
\hline & Yacaratiá & $25 / 02 / 13$ & Cubiertos & 49,0 & 10,70 & 78 & 53,70 & 16 & 0,96 \\
\hline
\end{tabular}

Los frutos del Guaporutí se cosecharon en noviembre de 2012 los cuales presentaron grados de coloración variable entre totalmente cubiertos y pintones (mitad del fruto con coloración). Del análisis del peso seco se estima un contenido de agua en el fruto que varía entre $82 \%$ para los frutos cubiertos y $86 \%$ en los frutos pintones.

El Siete Capotes se cosechó en enero, los frutos de esta especie presentan la particularidad de mantener el color verde ya que no cambian su coloración a medida que avanza la madurez de los mismos. Lo que se modifica con la maduración es la firmeza de la pulpa, la cual se hace notar al tacto (más o menos blando) siendo los frutos maduros mucho menos firmes. Respecto al tamaño los diámetros promedio de esta especie en este sitio fueron de
$25 \mathrm{~mm}$ tanto para frutos maduros e inmaduros. El peso fresco de los frutos maduros arrojaron un valor promedio de 7,70 $\mathrm{g}$ mientras que los verdes fue de 5,20 g promedio. En cuanto al peso seco, el peso promedio de los mismos fue de 1,50 y 1,60 g respectivamente, indicando un contenido de agua de entre $80,52 \%$ en los maduros y $69,24 \%$ en los inmaduros. El contenido de sólidos solubles en los frutos maduros fue de $14,75^{\circ}$ Brix mientras que en los inmaduros se registraron $12,50{ }^{\circ}$ Brix menos, en relación a la acidez los maduros presentaron un porcentaje de 4,40 y los inmaduros 3,74\% expresado como ácido cítrico por lo que se infiere que para esta especie no hay disminución de la acidez en el proceso de maduración, lo cual le confiere un sabor suavemente ácido, característico de los frutos de Siete Capotes 
que se asocia con el sabor del níspero y sus semillas al ser mordidas presentan un sabor parecido a alcanfor o eucaliptus.

El Aratikú fue la especie nativa más tardía ya que la cosecha de sus frutos se realizó en febrero. Una característica particular de esta especie es que sus frutos presentan una pulpa muy carnosa, comestible, de consistencia gelatinosa que rodea las semillas de gran tamaño. Esta característica particular se manifestó muy fuertemente en los frutos inmaduros por lo cual no fue posible la extracción de pulpa para el análisis de sólidos solubles y la acidez propiamente dicha. Los frutos de gran tamaño presentaron un diámetro promedio de $37 \mathrm{~mm}$ y con un peso promedio por fruto de hasta $30 \mathrm{~g}$. En cuanto al peso seco de los frutos verdes fue de $31 \mathrm{~g}$ lo que se corresponde con un $67,58 \%$ de agua en el fruto. Una de las características más interesantes de estos frutos fue el contenido de sólidos solubles ya que los mismos presentaron un valor promedio de $19,5{ }^{\circ}$ Brix, sumado a ello la acidez fue de $0,33 \%$ lo cual le confiere a los frutos del Aratiku un sabor muy dulce. Es una de las especies nativas con importancia frutícola más aceptadas en la zona por el gran tamaño de sus frutas y elevado contenido de sólidos solubles.

\section{Sitio B}

En el sitio de muestreo B del Salto Encantado la primer especie en cosecharse fue el Guaporutí en noviembre, frutos que presentaron una gran variación en la coloración de los mismos ya que en la misma fecha pudimos clasificarlos en cubiertos, verdes y pintones, en una proporción de 40\%, 40\% y $20 \%$ respectivamente.

El tamaño promedio de los frutos cubiertos fue de $14,28 \mathrm{~mm}$ y de los verdes y pintones fue de 13,33 $\mathrm{mm}$. Los pesos frescos promedios de los mismos fueron de 1,40 g y 1,30 g respectivamente. En cuanto al peso seco de los frutos cubiertos fue $0,30 \mathrm{~g}$ y de los verdes y pintones de 0,28 g. En ambos casos el contenido de agua fue muy similar arrojando un valor del $78,58 \%$ y $78,47 \%$. En lo que respecta a sólidos solubles presentes en los frutos cubiertos estos arrojaron un valor de $10,6^{\circ}$ Brix con una acidez del $0,25 \%$. En los frutos verdes y pintones los valores fueron de $8{ }^{\circ}$ Brix y $0,25 \%$ de acidez.
La acidez de los frutos no se modificó en el proceso de maduración y sólo los azúcares aumentaron confiriendo un sabor dulce y suave a los frutos cubiertos.

En el mes de enero se recolectaron los frutos de Ubajai en estado avanzado de maduración, presentando una coloración anaranjada fuerte. Se trata de una especie cuyas frutas presentaron un tamaño de $19 \mathrm{~mm}$ en promedio con un peso de $5 \mathrm{~g}$ aproximadamente. Del análisis de peso seco surge que estos frutos tuvieron $0,75 \mathrm{~g}$ con un contenido de agua del $85 \%$, por lo que estos frutos son los que presentaron el contenido de jugo más elevado de todos los analizados en este trabajo. Por su parte los sólidos solubles fueron de $8{ }^{\circ}$ Brix y una acidez bastante elevada del 3,78\%. Estas características se aprecian al gusto ya que los frutos de Ubajai presentaron poco sabor dulce y un marcado sabor ácido, lo que le confiere a estos frutos una característica distintiva comparada a las demás especies.

El Siete Capotes fue cosechado en Enero, encontrándose frutos con dos niveles de madurez, el tamaño de sus frutas fue de 30 $\mathrm{mm}$ en promedio y el peso fresco fue desde 5 a $12 \mathrm{~g}$, siendo los valores más elevados correspondieron a los frutos maduros. Los pesos secos fueron de 2,5 g en los maduros 10 que se corresponde a un $79,17 \%$ de agua, y en los frutos inmaduros el peso seco fue de $1,8 \mathrm{~g}$ y el contenido de agua del $68,97 \%$. Tanto los sólidos solubles como la acidez no presentaron cambios sustanciales en relación al estado de maduración de los frutos, presentando un contenido de azúcares de $13,5^{\circ}$ Brix para ambos estadios y una acidez de entre 4,98 a 5\% respectivamente. Estos resultados nos indicarían que el proceso de maduración no va acompañado de cambios en el contenido de sólidos solubles y acidez.

Otra especie que fructificó en febrero fue el Aratikú que dentro de las especies evaluadas fue una de la que presentó frutas de mayor tamaño en relación a las otras encontradas en este sitio. Se cosecharon frutos verdes y maduros pudiendo analizarse solamente los últimos por la difícil tarea de extracción de la pulpa en esta especie. El fruto presentó un tamaño promedio de $45,8 \mathrm{~mm}$ de diámetro los frutos verdes y un tamaño promedio de 
$46,8 \mathrm{~mm}$ los frutos cubiertos, mientras que el peso promedio que fue de $49,5 \mathrm{~g}$ y $69 \mathrm{~g}$ respectivamente. El valor promedio de peso seco de los frutos verdes fue de $16,32 \mathrm{~g}$, es decir, expresado como contenido de agua en el fruto arrojó un valor de $67 \%$, lo cual indica que se trata de un fruto con bajo contenido de agua. Los frutos cubiertos presentaron $19^{\circ}$ Brix y una acidez muy baja del $0,25 \%$, lo cual confirió al fruto un sabor muy dulce.

En el caso del Yacaratiá la cosecha de esta especie nativa se realizó en el mes de Febrero. Sus frutos fueron grandes arrojando un valor promedio de $53 \mathrm{~mm}$ de diámetro y un peso promedio de $49 \mathrm{~g}$ aproximadamente y se caracterizaron por poseer una coloración naranja fuerte. El peso seco registró un valor de $10,70 \mathrm{~g}$ lo que corresponde a un $78 \%$ de agua en el fruto y del análisis de los mismos se detectó un contenido de sólidos solubles de $16^{\circ}$ Brix y una acidez del $0,96 \%$. Al cortar los frutos se observó presencia de látex y que su forma y sabor se asemejan a las bayas del mamón o papaya, de allí deriva su nombre vulgar "Mamón de monte".

\section{Sitio C}

En este sitio de muestreo no se registraron estadíos de floración ni fructificación de los ejemplares. Se estima que esta condición se dio por ser éste un lugar en donde la radiación solar no penetra con facilidad y que presenta una elevada humedad relativa ambiente, sumado a que los árboles en su mayoría presentaban un estado sanitario comprometido.

\section{Conclusiones}

El presente trabajo permitió analizar la diversidad de especies nativas presentes en la flora misionera que tienen importancia frutícola para consumo humano.

Las especies frutícolas estudiadas en los sitios de muestreo son representativas de la flora de la Reserva y se estableció que las características físico químicas de calidad evaluadas en los frutos son similares en los diferentes sitios de muestreo, independientemente de la altitud.

El Aratikú se destaca por el alto contenido de azúcares, en todos los sitios de muestreo, lo cual le confiere un potencial valor de aprovechamiento.

Este trabajo es un aporte al conocimiento de la flora nativa con potencial frutícola presente en la provincia de Misiones, esperando que la información generada en esta investigación sea útil para profundizar en el aprovechamiento, conservación y conocimiento de nuestros recursos naturales.

\section{Agradecimientos}

A la Dirección de Áreas Naturales Protegidas, al Guardaparque José Luis Baecke, al Sr. Alberto Huber, al INTA EEA: Cerro Azul, Montecarlo, Aristóbulo del Valle y El Dorado, al Instituto de Botánica del Nordeste y al Instituto Línea Cuchilla.

\section{Bibliografía}

BERTOLINI, M. P. 1999. Plan de Manejo del Parque Provincial Salto Encantado del Valle del CuñáPirú. Ministerio de Ecología y Recursos Naturales Renovables. Gobierno de la Provincia de Misiones. $99 \mathrm{pp}$.

CIRIGNOLI, S., GALLIARI C. A., PARDIÑAS U. F. J., PODESTÁ D. H. \& ABRAMSON R. 2011. Mamíferos de la Reserva Valle del Cuña Pirú, Misiones, Argentina. Mastozoología Neotropical 18 (1): $25-43$.

LIGIER, H. D. 1990. Atlas de Suelo de la República Argentina. Tomo II. Provincia de Misiones. INTASAGYP.

LOPEZ, J. A., LITTLE, E. L., RITZ, G. F., ROMBOLD, J. S., HAHN, W. J. 1987. Árboles comunes del Paraguay. Nande Yvyra Mata Kuera. Colección e Intercambio de información. Servicio Forestal Nacional. Ministerio de Agricultura y Ganadería, Paraguay, $453 \mathrm{pp}$.

MARGALOT, J. A. 1985. Geografía de Misiones, Buenos Aires, $236 \mathrm{pp}$.

MÁRQUEZ, G., GIUDICE G. E. \& PONCE M. 2006. Pteridofitas de la Reserva "Valle del Arroyo Cuñá Pirú” (Misiones, Argentina). Darwiniana 44 (1): 108126.

ORTEGA TORRES, E., STUTZ DE ORTEGA, L., SPICHIGER, R. 1989. Flora del Paraguay. Noventa especies forestales del Paraguay. Serie especial número 3 , Conservatorio del Jardín botánico de la Ville de Ginebra, Editorial Missouri Botanical Garden, 300 pp. 
N. Pintaluba \& P. Alayón Luaces, Caracterización de frutas comestibles de especies nativas de Misiones

UNLP (Universidad Nacional de la Plata). 1992. Área de Ordenación Forestal, Dep. de Silvicultura, Fac. de Cs. Agrarias y Forestales; Informe Preliminar "Predio U.N.L.P., Prov. de Misiones".
1997. Grupo Interdisciplinario de la Facultad de Ciencias Naturales y Museo; Anteproyecto Área Protegida Cuñapirú, Aristóbulo del Valle, Pcia. de Misiones. Segundo Informe.

Original recibido el 31 de octubre de 2013; aceptado el 30 de noviembre de 2013. 
